\section{Rosmarie Barwinski: Resilienz in der Psychotherapie. Entwicklungsblockaden bei Trauma, Neurosen und frühen Störungen auflösen \\ (Klett-Cotta, Stuttgart, 2016)}

Jan-Erik Grebe (Zürich)

Dieses vielversprechende Buch beginnt den Leser schon mit seinem Klappentext zu fangen, in dem das «5-Punkte-Programm» der Autorin erwähn wird. Fünf Punkte, mit denen «krankmachende Prozesse in seelische Stärke» umgewandelt werden sollen - Resilienzförderung als Ziel der Psychotherapie. Der Rezensent fragte sich unwillkürlich, was er bisher für das Ziel von Psychotherapie hielt. Ähnlich verheissungsvoll geht es im Vorwort von Herrn Holderegger weiter, der beschreibt, dass der vorliegende Ansatz «als Schritt in Richtung der Entwicklung eines neuen Paradigmas für Psychotherapie(wissenschaft) verstanden werden» kann (S. 10). Entgegen dieser Ankündigung liesst sich das Buch angenehm unprätentiös. Die Autorin schafft es ihre, vornehmlich durch die Arbeiten Jean Piagets, Thomas Kesselrings und Gottfried Fischers angeregten Theorien trotz ihrer Komplexität mit Hilfe von einfachen aber gelungenen Fallvignetten zu verdeutlichen. Zentral für ihre Theorien ist hierbei das Konzept der Antinomien, das sie von Kesselring entlehnt und das sich vor allem durch das Vermischen verschiedener Ebenen, die ausdifferenziert und neu reintegriert bzw. konstruiert werden müssen, auszeichnet. Spürbar ist hierbei durchgehend, wie sehr der Autorin die Behandlung traumatisierter Menschen am Herzen liegt. Hier liegt eine deutliche Stärke des Buchs.

Das praktische Vorgehen nach dem Modell der Autorin wird von dieser ausführlich und verständlich erläutert: Zunächst werden die Abstraktionsebenen (die eine Verknüpfung zu Piagets Stufen der kognitiven Entwicklung darstellen) und damit das Funktionsniveau des Patienten bestimmt. Dies dient dazu, die Antinomien des Patienten zu erfassen, die im nächsten Schritt gelöst werden sollen. Gelegentlich erinnert dieser Punkt an das Bestimmen des Strukturniveaus des Patienten, worauf die Autorin jedoch nur am Rande eingeht. Sie unterscheidet dabei verschiedene Ätiologien, unter anderem Trauma-Folgeerkrankungen und frühe sowie neurotische Störungen (auf die auch in den letzten beiden Kapiteln des Buches näher eingegangen wird). Beim Lösen der Antinomien eines Patienten zeigt die Autorin ein pragmatisches und stets den Patienten stärkendes Vorgehen. Sätze wie: «Hinterfragt werden muss eine Heilungstheorie nur, wenn sie die Betroffenen krank macht» (S. 169) sind nicht selten.

Im nächsten Schritt greift das «5-Punkte-Programm», in dem das problematische Verhalten ausfindig gemacht und zu diesem ein Gegenpol gefunden werden soll. Diese Vorstellung der allen Dingen inne liegenden Gegenpole entnimmt die Autorin den Theorien Fischers, in denen diese zu verstehen sind als «aufgespaltene Polaritäten», die «wieder verbunden werden» müssen. Oder wie die Autorin schreibt: "Auf Nähe bezogen bedeutet dies: Gesunde Nähe ist nur möglich, wenn ich auch weggehen kann» (S. 149). Aus diesem problematischen Verhalten und dem nicht minder problematischen Gegenpol sollen im nächsten Schritt die positiven Komponenten extrahiert, dann verbunden und abschliessend die Eigenaktivität des Patienten gefördert werden, die es ihm ermöglichen soll, die beiden positiven Komponenten auch umzusetzen. Trotz der unterstrichenen Notwendigkeit der Transferleistung betont die Autorin, dass «langfristige, innerseelische Einsichtsprozesse» (S. 161) vorausgehen müssen, um die Resilienz zu fördern und ein reines Verhaltenstraining dies nicht liefern könne.

Im letzten Schritt, der das traumakompensatorische Schema von Fische und Riedesser als Grundlage hat, geht es der Autorin um die «Stärkung eigener Bewältigungsstrategien». Vor allem hier spürt man die Andersartigkeit des Ansatzes der Autorin und seine Wurzeln in der Behandlung von Trauma-Folgeerkrankungen: Die Fähigkeit des Patienten aus Eigenaktivität jenseits von Anlage und Umwelt Resilienz aufzubauen, soll gefördert werden. Das vom Patienten für sich selbst entwickelte kompensatorische Schema wird ihm nicht genommen, nicht überschrieben mit Übungen, sondern als Überlebensstrategie gewürdigt und mit dem Patienten so modifiziert, dass es seine Schutzfunktion erfüllt, ohne den Patienten zu schädigen. Das Schema wird hierfür mit dem Patienten besprochen: Es wird validiert, seine Nachteile werden aufgezeigt und anschliessend gestärkt und in seiner Funktion differenziert. Die Autorin schafft es hierbei, einen die Würde des Patienten wahrenden Blick zu vermitteln, der die Handlungsfähigkeit des Patienten unterstreicht, anstatt bei den Defiziten zu verharren.

Neben diesen sehr begrüssenswerten Ansätzen stellt sich gelegentlich die Frage, an welche Zielgruppe sich das Buch richtet. So werden zwar zahlreiche psychodynamische Konzepte verwendet, jedoch werden diese häufig auf eine Art und Weise vorgestellt, die sich eher an nicht psychodynamisch arbeitende TherapeutInnen zu richten, vielmehr Schulen übergreifend gedacht zu sein scheint. Stellenweise mangelt es diesen so an überzeugender Strahlkraft. Daher 
wirkt es gelegentlich zu sehr nach dem Versuch, die Theorien der Autorin in das Psychodynamische einzubetten. Etwas mehr Ausdifferenzierung der verschiedenen, in diesem Fall therapeutischen Ebenen, die die Autorin bei den Antinomien beschreibt, wäre auch hier wünschenswert gewesen und hätte das Buch nicht weniger lesenswert gemacht. Als bedauerlich empfand der Rezensent den Mangel an Bezug der Theorien seitens der Autorin zu verschiedenen, bereits bestehenden theoretischen Konzepten, wie etwa der operationalisierten psychodynamischen Diagnostik oder auch den Theorien der strukturbezogenen Psychotherapie nach Gerd Rudolf. Zu guter Letzt könnte der didaktische Aufbau des Buches und der Methode, in ihrer Manualhaftigkeit, den einen oder anderen vom Lesen abschrecken - was schade wäre. 